\title{
Case report on a rare entity-Kikuchi Fujimoto Disease (KFD) in a person with underlying Systemic Lupus Erythematosus (SLE)
}

\begin{abstract}
Kikuchi-Fujimoto disease (KFD) is a rare benign condition of necrotizing histiocytic lymphadenitis. The case of a 31-year-old gentleman who presented with fever, weight loss, and a single tender right-sided cervical lymph node is described here. Symptomatic treatment was provided initially, but the course was complicated with an episode of aseptic meningitis and admission to the medical intensive care unit (MICU). The Liver enzymes and lactate dehydrogenase $(\mathrm{LDH})$ were also found to be very high during this period but returned to baseline. He was also found to have oral ulcers, pleural effusion, positive serology for lupus, and pancytopenia with lymphopenia and autoimmune hemolytic Anemia. The entire illness lasted for about six weeks. Although tissue diagnosis was delayed because of the MICU admission, KFD was finally diagnosed after cervical lymph node biopsy. Cases of KFD has been reported widely, but most of them had an uneventful course. We believe that the eventful course and the presence of underlying lupus make this case worth reporting. Take away lesson from this case is that tissue diagnosis should not be delayed in persons presenting with generalized or localized lymphadenopathy associated with systemic symptoms and along with common conditions rare entities like KFD should be considered especially if the patient does not respond to routine treatment.
\end{abstract}

Volume 10 Issue 4 - 2020

\author{
Sreethish Sasi, Nadia Karim Bakhsh, Raza Ali \\ Akbar \\ Department on Internal Medicine, Hamad General \\ Hospital,Qatar
}

Correspondence: Sreethish Sasi, Medical Resident, Department of Internal Medicine, Hamad Medical Corporation, Qatar,Tel +974- 50290089, Email Ssasi@hamad.qa

Received: July 24, 2020 | Published: August 17, 2020

Keywords: necrotizing lymphadenopathy, aseptic meningitis, systemic lupus erythematosus

Abbreviations: KFD, kikuchi-fujimoto disease; MICU, medical intensive care unit; LDH, lactate dehydrogenase; SLE, systemic lupus erythematosus; TB, tuberculosis; CRP, c-reactive protein; ESR, erythrocyte sedimentation rate; CMV, cytomegalovirus; EBV, epstein-barr virus; HIV, human immunodeficiency virus; PCR, polymerase chain reaction; ANA, antinuclear antibody; RF, rheumatoid factor; CT, computed tomography; MRI, magnetic resonance imaging; EEG, electroencephalogram; CNS, central nervous system; CSF, cerebrospinal fluid; ESR, erythrocyte sedimentation rate

\section{Introduction}

When a patient presents with a neck mass associated with non -specific systemic signs and symptoms, the initial differentials include the more common ones such as lymphoma, tuberculosis, and systemic lupus erythematosus (SLE). However, rarer conditions such as KFD must still be considered, especially when the patient's condition fails to abate. About $40 \%$ of the patients with KFD were initially misdiagnosed to have malignant lymphoma and were unnecessarily treated with chemotherapy. This was evident in a case series by Dorfman et al. ${ }^{1}$ Because of this illness's self-limiting nature, early diagnosis helps avoid unnecessary treatment and lessens the emotional stress of the patient and his family. Herein we discuss a case of a young man who presented with a neck mass, which was not attributable to the more common causes.

\section{Case presentation}

A 31-year-old South-East Asian gentleman, who was a cigarette smoker for the past six years (3 pack-years) and occasional alcoholic, with no significant history of medical illness in the past and no relevant family history presented with a ten-day history of high-grade fever with rigor and chills, dry cough, night sweats, anorexia, weight loss (8kilograms in 2 months), and painful right-sided neck swelling. There were no other complaints. He had been previously fit and well and was not on any medication. On examination, he was lethargic and febrile but otherwise looked well and hemodynamically stable. A significant finding was a sizeable, diffuse, and tender non-fluctuant swelling deep to the lateral border of right sternocleidomastoid at levels $2-3$, of size approximately $4 \times 4 \mathrm{~cm}$. There were also multiple small non- tender cervical lymph nodes at levels $2,3,4$, and 5 but none in the axilla or inguinal region and no palpable hepatosplenomegaly. A diffuse erythematous maculopapular non-blanching rash was noted on the backside of his trunk below the neck. It was non-itchy and non-tender.

Blood tests revealed pancytopenia with normocytic normochromic anemia, leucopenia with significant neutropenia, and low platelets (Table 1). Serum LDH was more than $1000 \mathrm{U} / \mathrm{L}$ with low serum haptoglobin $(<4$ milligrams per deciliter $)$, indicating intravascular hemolysis. Serum electrolytes were normal, but liver enzymes were significantly elevated (Table 1). C-Reactive protein (45milligrams per liter) and Procalcitonin (0.29nanograms per milliliter) were mildly elevated. Electrocardiogram was normal, but chest radiographs showed bilateral pleural effusion (Figure 1). Peripheral smear showed mild normocytic normochromic anemia with few spherocytes and moderate rouleaux formation, leukopenia with neutropenia, and many pleomorphic, reactive and atypical lymphocytes (with irregular nuclei $\&$ abundant basophilic cytoplasm). Platelets were moderately reduced. Serological tests for common viral diseases such as cytomegalovirus (CMV), Epstein-Barr virus (EBV), Human Immunodeficiency Virus (HIV), and hepatitis; blood, urine, and stool cultures were negative. Sputum cultures grew respiratory tract flora and were negative for 
acid-fast bacilli. All workups for tuberculosis (TB) were negative, including quantiferon, sputum acid fast bacilli smear, culture and polymerase chain reaction (PCR). Antinuclear antibody (ANA), Anti - Smith's Antibody, and Anti RNP antibodies were positive, while
Rheumatoid Factor (RF) was negative. Serum C3 and C4 were low. Flow cytometry from Bone Marrow biopsy and Lymph node aspiration biopsy was found to be negative for lymphoma and leukemia.

Table I Table showing results of relevant laboratory tests on initial presentation and during follow-up

\begin{tabular}{|c|c|c|c|c|}
\hline \multirow[b]{2}{*}{ Detail } & \multicolumn{3}{|l|}{ Results } & \multirow[b]{2}{*}{ Normal Range } \\
\hline & During illness & $\begin{array}{l}\text { During follow up after } \\
\text { 2months }\end{array}$ & $\begin{array}{l}\text { During follow up after } \\
\text { I year }\end{array}$ & \\
\hline White Blood Cells $\left(\times 10^{3} / \mu \mathrm{L}\right)$ & 3 & 5.1 & 4.1 & $4-10$ \\
\hline Absolute Neutrophil count (ANC) \% & 27.5 & 34 & 58.5 & $55-70$ \\
\hline Lymphocytes \% & 52.5 & 54.5 & 33.7 & $20-40$ \\
\hline Monocytes \% & 19.5 & 6.9 & 6.3 & $2-8$ \\
\hline Eosinophils \% & 0.2 & 5 & I & $1-4$ \\
\hline Basophils \% & 0.2 & 0.6 & 0.5 & $0.5-1$ \\
\hline Platelets $\left(\times 10^{3} / \mu \mathrm{L}\right)$ & 69 & 208 & 145 & $150-400$ \\
\hline Red Blood Cells $\left(\times 10^{3} / \mu \mathrm{L}\right)$ & 2.8 & 4.9 & 4.9 & $4.5-5.5$ \\
\hline Haemoglobin (gm/dL) & 8.1 & 13.8 & 14.6 & $12.0-15.0$ \\
\hline Urea (mmol/L) & 2.5 & 2.5 & 3.6 & $2.5-6.7$ \\
\hline Creatinine $(\mu \mathrm{mol} / \mathrm{L})$ & 51 & 67 & 73 & $50-98$ \\
\hline Total Bilirubin $(\mu \mathrm{mol} / \mathrm{L})$ & 37 & 6 & 9 & $3.4-20.5$ \\
\hline Alkaline Phosphatase (U/L) & 101 & 47 & 54 & $40-150$ \\
\hline Alanine Amino Transferase (U/L) & 350 & 17 & 17 & $0-55$ \\
\hline Aspartate Amino Transferase (U/L) & 932 & 18 & 14 & $5-34$ \\
\hline Lactate Dehydrogenase (LDH - (U/L)) & 1458 & 192 & $|5|$ & $135-225$ \\
\hline Serum C3 (mg/dl) & 51.7 & 78.5 & 50.9 & $90-180$ \\
\hline Serum C4 (mg/dl) & 8.6 & 11.5 & 7 & $10-40$ \\
\hline $\mathrm{CRP}(\mathrm{mg} / \mathrm{L})$ & 27 & 5 & 2.3 & $0-5$ \\
\hline
\end{tabular}

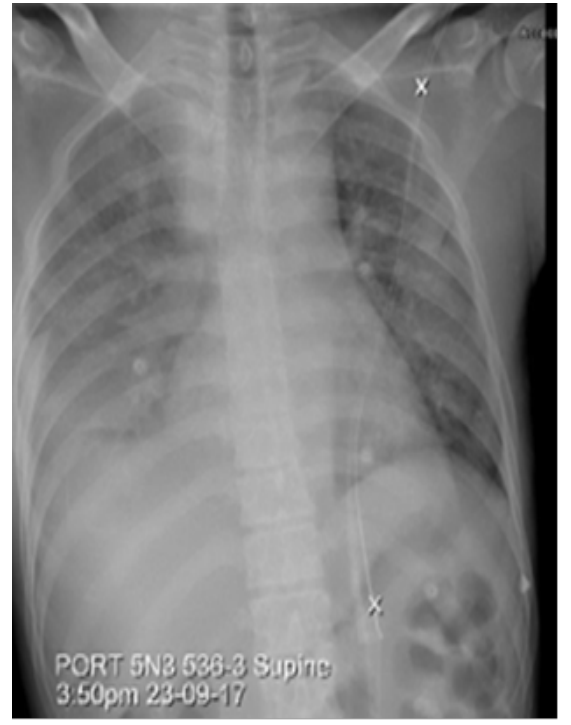

Figure I Chest X-Ray on admission showing bilateral pleural effusion.
A CT scan of the neck was done due to clinical suspicion of a retropharyngeal abscess, which showed multiple enlarged necrotic lymph nodes on the right side of the neck (Figure 2). His initial management consisted of empiric antibiotics (Amoxicillin + Clavulanic Acid) and regular paracetamol, while the results of further tests were awaited. Subsequently, there was a reduction in the size and tenderness of the cervical lymph nodes, but there were persistent intermittent temperature spikes. Ten days after the initial presentation, he developed an episode of acute confusion with a drop in saturation and abnormal movements. He also complained of a severe headache, but there was no nuchal rigidity. Kernig's and Brudzinski's signs were also negative. Computed Tomography (CT) head, Magnetic Resonance Imaging (MRI) Brain, and electroencephalogram (EEG) were unremarkable. In MRI, there was no evidence of Lupus cerebritis or central nervous system (CNS) vasculitis. Lumbar punctures showed high protein, low sugar, and 22 white blood cells with 92\%lymphocytes. Cerebrospinal fluid (CSF) culture and Viral PCR were negative, and hence this followed a pattern of aseptic meningitis. He was empirically treated with acyclovir and meropenem for two days, which were later discontinued. He made a spontaneous recovery from CNS symptoms over the next eight days. Excisional biopsy of the 
right cervical Level 3 and Level 5 Lymph nodes confirmed a diagnosis of Kikuchi-Fujimoto disease (KFD). Histological analysis showed histiocytic granulomatous infiltration with widespread necrosis of the lymph node extending beyond its capsule into the surrounding fat. There were areas of xanthomatous cells and areas of partial coagulative necrosis with some karyorrhexic debris containing a variety of cells, including histiocytes, plasmacytoid monocytes, some immunoblasts, and some small and large lymphocytes. Immunohistochemistry was also consistent with this diagnosis.

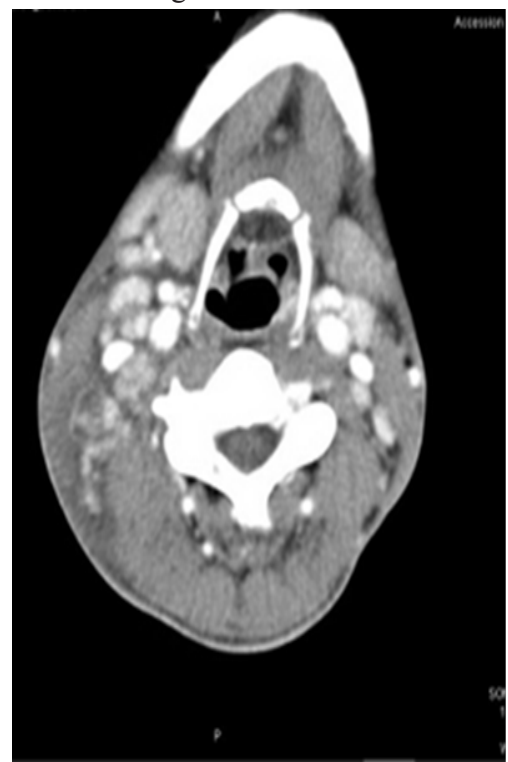

Figure 2 A CT scan of the neck showing multiple enlarged necrotic lymph nodes on the right side of the neck.

His illness's total duration was around 5 to 6 weeks at the end of which he was entirely asymptomatic. But given his positive ANA, anti-smith antibody, low C3 - C4, pancytopenia, intravascular hemolytic anemia, oral ulcers, and pleural effusion, he was diagnosed to have underlying SLE and was discharged home on oral azathioprine and prednisolone. His maculopapular skin rash also resolved in 4 weeks without any treatment. At follow-up, he reported no symptoms, remained well, and there were no abnormalities on clinical examination.

\section{Discussion}

Kikuchi Fujimoto Disease is a rare, benign histiocytic necrotizing lymphadenitis. It is most commonly reported in Asians, and etiology is unclear. Associations have been found with EBV, human herpes virus 6 and 8, and SLE. ${ }^{2}$ Most commonly affects young people under 30 years of age, with equal incidence in both sexes. ${ }^{3}$ Usually presents as painless cervical lymphadenopathy with constitutional symptoms. Associated hepatomegaly, leukopenia, increased erythrocyte sedimentation rate (ESR), increased liver enzyme levels. Most cases of KFD have a single large posterior cervical lymph node, as seen in our patient. ${ }^{4}$ Preceding flu-like illness occurs in half of the KFD cases, and clinical resolution occurs in one to two months in most cases. ${ }^{5}$ Typical lymph node biopsy findings include necrosis, karyorrhectic debris, and the presence of the typical cell types, namely crescentic histiocytes, and plasmacytoid monocytes. ${ }^{6}$ Extra nodal manifestations of KFD include maculopapular skin lesions, morbilliform rash, nodules, or urticaria, which resolve in a few weeks, hepatosplenomegaly, aseptic meningitis (with negative Kernig's and
Brudzinski's sign), and arthritis. ${ }^{7}$ Closest differentials of KFD are malignant lymphoma and SLE. Treatment of KFD is symptomatic and supportive, spontaneous recovery is usual, while the latter two conditions require prompt specific therapies. ${ }^{8}{ }^{89} \mathrm{KFD}$ can mimic SLE both clinically and histologically. Many cases of KFD has been reported in persons with underlying SLE. T lymphocytes predominate in Kikuchi disease, whereas B lymphocytes predominate in SLE. ${ }^{9}$ Indications for steroid therapy in KFD include features of neurologic involvement such as aseptic meningitis or cerebellar ataxia, hepatic involvement such as elevated LDH, and severe lupus-like syndrome with positive ANA. ${ }^{10}$

\section{Conclusion}

KFD is uncommon but should feature in a list of differential diagnoses of tender cervical lymphadenopathy. Its treatment differs significantly from other similar conditions such as SLE, lymphoma, and TB. Lymph node biopsy will aid accurate diagnosis, but if confusion with SLE occurs, differentiation can be made with the aid of blood tests for complement levels, amongst others.

\section{Acknowledgments}

The authors would like to acknowledge Dr. Dabia Al Mohanadi, Program Director, Internal Medicine Residency Program of Hamad Medical Corporation for scientific support.

\section{Statement of ethics}

The case was approved by the Medical Research Center at Hamad Medical Corporation. Written informed consent was given by the patient to publish his case information, images and details.

\section{Funding}

This study was funded by Qatar National Library.

\section{Conflicts of interest}

The authors have no conflicts of interest to declare.

\section{References}

1. Dorfman RF, Berry GJ. Kikuchi’s histiocytic necrotizing lymphadenitis: an analysis of 108 cases with emphasis on differential diagnosis. Semin Diagn Pathol. 1988;5(4):329-345.

2. Singh JM, Shermetaro CB. Kikuchi-Fujimoto Disease in Michigan: A Rare Case Report and Review of the Literature. Clin Med Insights Ear Nose Throat. 2019;12:1179550619828680.

3. Jung IY, Ann HW, Kim JJ, et al. The incidence and clinical characteristics by gender differences in patients with Kikuchi-Fujimoto disease. Medicine (Baltimore). 2017;96(11):e6332.

4. Poulose V, Chiam P, Poh WT. Kikuchi's disease: a Singapore case series Singapore Med J. 2005;46(5):229-232.

5. Mahajan T, Merriman RC, Stone MJ. Kikuchi-Fujimoto disease (histiocytic necrotizing lymphadenitis): report of a case with other autoimmune manifestations. Proc (Bayl Univ Med Cent). 2007;20(2):149 151.

6. Supari D, Ananthamurthy A. Kikuchi-fujimoto disease: a study of 24 cases. Indian J Otolaryngol Head Neck Surg. 2014;66(1):69-73.

7. Kataria R, Rao P, Kachhawa D, et al. Kikuchi-Fujimoto Disease, the Masquerading Menace: A Rare Case Report. Indian J Dermatol. 2016;61(3):348. 
8. Boddu P, Mohammed AS, Annem C, et al. SLE and Non-Hodgkin's Lymphoma: A Case Series and Review of the Literature. Case Rep Rheumatol. 2017;2017:1658473.

9. Baenas DF, Diehl FA, Haye Salinas MJ, et al. Kikuchi-Fujimoto disease and systemic lupus erythematosus. Int Med Case Rep J. 2016;9:163-167.
10. Yalcin S, Toprak SK, Erismis B, et al. Management of kikuchifujimoto disease using glucocorticoid: a case report. Case Rep Med. 2011;2011:230840. 